\title{
Susceptibility vessel sign predicts poor clinical outcome for acute stroke patients untreated by thrombolysis
}

\author{
HUIQIN LIU ${ }^{1 *}$, WENLI MEI ${ }^{1 *}$, YUE HUANG $^{1}$, YONGLI LI ${ }^{2}$, \\ ZUZHI CHEN ${ }^{1}$, DONGDONG LI $^{3}$, $\mathrm{HONG} \mathrm{YE}^{1}$ and JIEWEN ZHANG ${ }^{1}$

\begin{abstract}
Departments of ${ }^{1}$ Neurology and ${ }^{2}$ Radiology, People's Hospital of Zhengzhou University, Zhengzhou, Henan 450003; ${ }^{3}$ Department of Neurology, The First Affiliated Hospital of Xinxiang Medical University, Xinxiang, Henan 453100, P.R. China
\end{abstract}

Received October 4, 2016; Accepted June 2, 2017

DOI: $10.3892 /$ etm.2017.5195

\begin{abstract}
The location and length of the susceptibility vessel sign (SVS) predicts poor outcome for patients having received reperfusion therapy. The aim of the present study was to assess the predictive value of SVS regarding the clinical outcome for patients untreated with thrombolysis. A retrospective study on acute stroke patients who underwent multimodal magnetic resonance imaging within 3 days from the onset of symptoms was performed. None of the patients had received thrombolysis therapy. The presence, location and length of the SVS were assessed. Uni- and multivariate analyses were used to examine the association between SVS and clinical outcome. A total of $43 \mathrm{SVS}+$ and 73 SVS- patients were included in the study. A modified Rankin Scale (mRS) of $\leq 2$ at 3 months was determined in $41.9 \%$ of patients in the SVS+ group and $79.4 \%$ in the SVS-group $(\mathrm{P}<0.001)$. Multivariate analysis revealed that the presence of SVS was an independent parameter to predict $\mathrm{mRS}>2$ at 3 months (odds ratio, 3.390; $95 \%$ confidence interval, 1.122-10.240; $\mathrm{P}=0.030$ ). For patients with SVS+ status, the location and length of the SVS were not independent predictors of the clinical outcome. In conclusion, the presence of SVS may predict poor clinical outcome for acute stroke patients untreated with thrombolysis.
\end{abstract}

Correspondence to: Dr Jiewen Zhang, Department of Neurology, People's Hospital of Zhengzhou University, 7 Weiwu Road, Zhengzhou, Henan 450003, P.R. China

E-mail: zjw1503@126.com

${ }^{*}$ Contributed equally

Abbreviations: SVS, susceptibility vessel sign; MRI, magnetic resonance imaging; MCA, middle cerebral artery; SWI, susceptibility-weighted imaging; t-PA, tissue-plasminogen activator; DWI, diffusion-weighted imaging; MRA, magnetic resonance angiography; NIHSS, National Institutes of Health Stroke Scale; mRS, modified Rankin Scale; CBS, clot burden score; TOAST, Trial of ORG 10172 in Acute Stroke Treatment

Key words: ischemic stroke, magnetic resonance imaging, outcome, susceptibility vessel sign, susceptibility-weighted imaging

\section{Introduction}

Thrombosis is the most common cause of vascular occlusion in ischemic stroke. Timely and accurate assessment of the location, clot burden and size of the thrombus is of great significance for guiding and monitoring the clinical treatment. The hyperattenuated middle cerebral artery (MCA) sign on computed tomography (CT) was the first imaging method for directively detecting intra-arterial thrombus, but its diagnostic sensitivity is only $22.6-40 \%(1,2)$, limiting its diagnostic value. In this century, the susceptibility vessel sign (SVS) of the MCA on gradient-recalled echo (GRE) imaging was demonstrated to be well correlated with the hyperdense MCA sign on $\mathrm{CT}$, and had a high sensitivity varying in between 40 and $100 \%$ to detect intra-arterial thrombus $(3,4)$. The GRE SVS reflected thrombus composed of deoxyhemoglobin produced by desaturation of oxyhemoglobin. Thus, GRE SVS is present in older thrombi. In hyperacute clot cases, the main component may still be oxyhemoglobin, and such emboli would not be identified as GRE SVS on T2* imaging (4). The SVS on susceptibility-weighted imaging (SWI) is based on a similar theory and also has a high sensitivity and specificity to detect the intra-arterial thrombus in acute stroke (5). Numerous studies have assessed the predictive value of the characteristics of SVS regarding the outcome of patients who had received tissue-plasminogen activator (t-PA) administration or endovascular treatment, and found that the location, clot burden and morphology of the SVS may predict the absence of early recanalization or poor outcome (6-12). However, none of these studies has focused on SVS of patients untreated with reperfusion therapy. Therefore, the present study used SWI to explore the association of SVS with the clinical outcome for acute ischemic stroke patients not subjected to thrombolysis.

\section{Materials and methods}

Patients. A retrospective study of patients with acute ischemic stroke who were admitted to the People's Hospital of Zhengzhou University (Zhengzhou, China) from January 2013 to May 2015 was performed. Patients were enrolled if they met the following inclusion criteria: i) Acute cerebral infarction confirmed by diffusion-weighted imaging (DWI); ii) cerebral infarction distributed in the anterior circulation territory and 
non-lacunar infarcts; and iii) a stroke magnetic resonance (MR) imaging protocol including DWI, SWI and MR angiography (MRA) performed within 3.0 days of the onset of symptoms. All patients were treated with anti-platelet or anti-coagulant therapy. Patients who had undergone thrombolytic therapy or endovascular treatment were excluded. Stroke severities were evaluated by the National Institutes of Health Stroke Scale (NIHSS) on admission. A favorable outcome was defined as having a modified Rankin Scale (mRS) of 0-2 at 3.0 months after stroke, while a poor outcome was defined as having an mRS >2 (13). The Institutional Review Board of the People's Hospital of Zhengzhou University (Zhengzhou, China) approved the present study; all patients provided their informed consent for the entry of their data/images into the stroke database.

The following baseline demographic and clinical information on all patients was retrieved: Age, sex, time from onset to treatment, time from onset to imaging, vascular risk factors, atrial fibrillation, blood pressure before treatment, fasting blood glucose and stroke etiology. Vascular risk factors were identified as follows: i) Hypertension, a history of using anti-hypertensive agents, a systolic blood pressure $\geq 140 \mathrm{~mm}$ $\mathrm{Hg}$ or a diastolic blood pressure $\geq 90 \mathrm{~mm} \mathrm{Hg}$ at hospital discharge; ii) diabetes mellitus, use of hypoglycemics, random glucose level $\geq 11.1 \mathrm{mmol} / 1$, or glycosylated hemoglobin $>6.4 \%$ on admission; iii) hyperlipidemia, use of anti-hyperlipidemic agents, or serum cholesterol level $>220 \mathrm{mg} / \mathrm{dl}$; or iv) hyperhomocysteinemia, serum homocysteine level $>15 \mu \mathrm{mol} / 1$. Stroke etiology was determined at hospital discharge using the Trial of ORG 10172 in Acute Stroke Treatment (TOAST) criteria: i) Large-artery atherosclerosis; ii) cardioembolism; or iii) a different determined or undetermined etiology of stroke (14). Small-vessel occlusion was excluded to ensure that the infarction was derived from the large blood vessels.

MRI protocol. Multimodal MRI was performed on a Siemens Magnetom Trio, Tim 3.0 T system (Siemens AG, Munich, Germany) using commercially available hardware and software. The following sequences were obtained: DWI [repetition time (TR), 3,100 msec; echo time (TE), $82 \mathrm{msec}$; b-value, $1,000 \mathrm{sec} / \mathrm{mm}^{2}$; acquisition matrix, 128x128; field of view (FOW), $240 \mathrm{~mm}$; section thickness, $6.0 \mathrm{~mm}$; section gap; $1.2 \mathrm{~mm}$; duration; $48 \mathrm{sec}$ ). SWI (TR/TE/flip angle, $27 \mathrm{msec} / 20 \mathrm{msec} / 15^{\circ} \mathrm{C}$; FOV, $240 \mathrm{~mm}$, acquisition matrix, 384x32; section thickness, $3.0 \mathrm{~mm}$; duration, $3.0 \mathrm{~min} 27 \mathrm{sec}$ ). Three-dimensional time-of-flight MRA (TR/TE/flip angle, $20 \mathrm{msec} / 3.2 \mathrm{msec} / 18^{\circ}$; FOV, $230 \mathrm{~mm}$, acquisition matrix, 256x256; section thickness, $0.9 \mathrm{~mm}$; duration, $3.0 \mathrm{~min} 6.0 \mathrm{sec}$ ).

Imaging analysis. All images were collected from the picture archiving and communication system of the hospital as Digital Imaging and Communications in Medicine-format data and imported to syngo fastView version 02184790 (Siemens AG) for analysis. The MRA rating, diffusion lesion volumes and SVS features were independently assessed by two neurologists (H.L. and W.M.), who were blinded to patient information, except for the clinical history of ischemic stroke. Any disagreement was decided by consultation with a neuroradiologist (Y.L.). The SVS was defined as a hypointense signal on SWI within a vascular cistern, exceeding the size of the homologous contralateral arterial diameter (4). The location and the length of SVS were also assessed. Proximal M1 SVS was defined as an SVS at the origin of the M1, distal M1 SVS was defined as any SVS not including the origin of the M1 and distal MCA SVS was considered as an SVS further away from M1 (Fig. 1) (8). The thrombus length was measured as the whole extension of the thrombus on SWI (15). All areas of diffusion were measured by drawing regions of interest around the lesions by manual editing on a slice-by-slice basis. DWI lesion volumes were identified as the regions with an intensity higher than that in the contralateral lobe by 3.0 standard deviations (SDs). Volumes were calculated by multiplying overall outlined lesion areas by the slice thickness. MRA was rated by using the modified thrombolysis in cerebral infarction (TICI) score (16). The vascular stenosis site was rated using the following assessment: 1, internal carotid artery (ICA); 2, proximal MCA, including M1 segment of the MCA; 3, distal MCA, M2 segment of the MCA or further branches.

Statistical analysis. First, all patients were classified into 2 groups based on the presence of SVS. Clinical, radiological and laboratory findings were compared between the 2 groups. Values are expressed as the mean \pm SD, median (25th, 75th percentile) or $n(\%)$. Statistical comparisons were performed by independent samples t-test for normally distributed continuous variables, the Mann-Whitney $U$ test for abnormally distributed continuous or ordinal variables, and the $\chi^{2}$ test for categorical variables. Furthermore, all patients were divided into 2 groups based on a good or poor outcome at 3 months, and the clinical, radiological and laboratory findings were compared between the 2 groups. Multivariate logistic regression analysis was used to identify independent factors associated with poor outcome. Variables identified on univariate analyses with $\mathrm{P}<0.05$ were entered into the multivariate analysis. Results are expressed as adjusted odds ratios (ORs) and the corresponding $95 \%$ confidence intervals (CIs). In addition, for patients with SVS, univariate and multivariate analyses were performed to determine whether the location and length of SVS were independent parameters associated with poor outcome. Statistical significance was set at $\mathrm{P}<0.05$. All statistical analyses were performed using SPSS version 21 (IBM Corp., Armonk, NY, USA).

\section{Results}

Patient characteristics. The 127 consecutive patients who met the inclusion criteria were enrolled in the present study. A total of 11 patients were excluded due to the following reasons: 6 were lost to follow-up, 4 had received thrombolytic therapy and 1 had received endovascular treatment. Of the 116 remaining patients who were included in the study, $68.1 \%$ were men, the mean age of all patients was $59.91 \pm 13.86$ years, 20 were confirmed to have ICA stenosis, 64 had MCA M1 stenosis, 21 had stenosis at M2 or further branches of the MCA and 11 had normal arteries. Stroke etiology according to the TOAST classification was large artery atherosclerosis $(n=98)$, cardioembolism $(n=10)$ and other determined and undetermined etiologies $(n=8)$. Sixty patients had infarction in the right cerebral hemisphere and 56 had infarction in left hemisphere (Table I). 


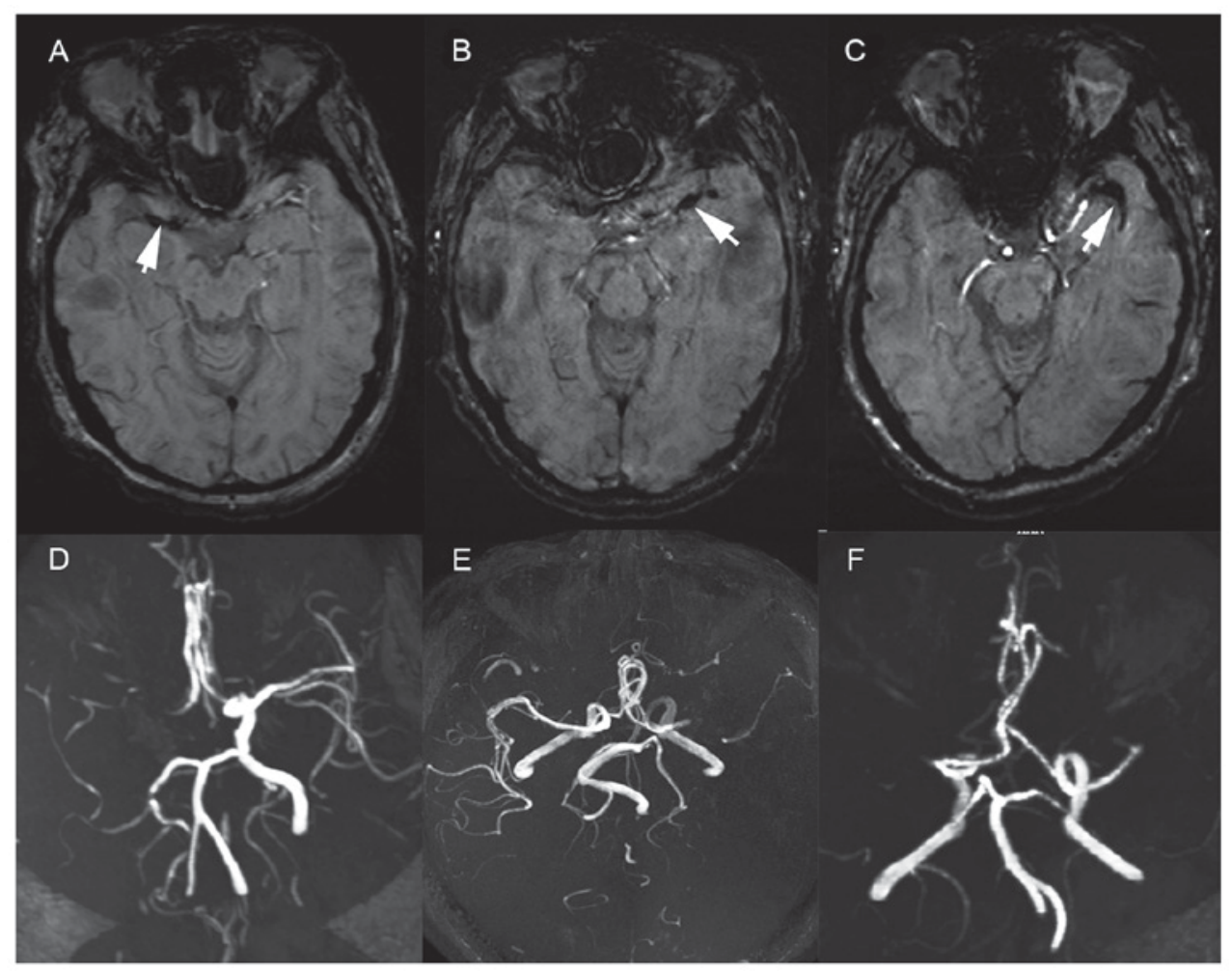

Figure 1. Representative images of SVS on susceptibility-weighted imaging (white arrows) and the corresponding MRA in 3 patients. (A) SVS located at the proximal M1; (B) SVS located at the distal M1; (C) SVS located at the M2 (distal middle cerebral artery); (D-F) Corresponding MRA images of the patients with an SVS in A, B and C, respectively. SVS, susceptibility vessel sign; MRA, magnetic resonance angiography.

Characteristics of SVS (+) vs. SVS (-) patients. SVS were seen in 43 of the 116 patients. The patients' clinical characteristics and imaging findings according to the SVS presence are listed in Table I. Compared with those in the SVS(-) group, patients with SVS(+) had a higher systolic pressure $(149.72 \pm 23.59$ vs. $137.74 \pm 17.29 \mathrm{mmHg} ; \mathrm{P}=0.005)$, lower TICI scores [0 (0-1) vs. $2(1-3) ; \mathrm{P}<0.001]$, larger infarction areas [65.08 (21.43-140.96) vs. $12.96(4.71-33.52) \mathrm{ml} ; \mathrm{P}<0.001]$ and higher NIHSS scores on admission [12 (5-20) vs. 5 (2-8); $\mathrm{P}<0.001]$. The TOAST classification of patients with SVS $(+)$ identified all of them as having had cardioembolic stroke or large artery atherosclerosis. Of the $43 \mathrm{SVS}(+)$ patients, $18(41.9 \%)$ had favorable outcomes, while $58(79.5 \%)$ of the 73 SVS(-) patients achieved good outcomes $(\mathrm{P}<0.001)$.

Factors associated with poor outcome in patients with acute ischemic stroke. Table II presents univariate analysis of the variables between patients with and without favorable outcome. Patients with a poor outcome were older than those with a good outcome $(65.35 \pm 12.04$ vs. $57.35 \pm 13.96$ years; $\mathrm{P}=0.002)$, more of them had atrial fibrillation (15 vs. $1.3 \%$; $\mathrm{P}=0.003)$ and the presence of SVS (62.5 vs. $23.7 \% ; \mathrm{P}<0.001)$ than patients with a good outcome. The DWI lesion volumes were larger [39.27 (17.24-154.47) vs. 13.53 (6.60-44.69) ml; $\mathrm{P}=0.001]$ and the NIHSS scores on admission were higher [13.5 (7.25-22.75 vs. $5(2-8)$; $\mathrm{P}<0.001]$ in patients with a poor outcome than in those with a good outcome. The TICI scores were lower in patients with a poor outcome [1 (0-2) vs. $2(0-2)$; $\mathrm{P}=0.043]$. Compared with patients with favorable outcome, the TOAST classification of patients with poor outcome was more likely to be large artery atherosclerosis or cardioembolism $(\mathrm{P}=0.029)$. Finally, seven factors including age, atrial fibrillation, the presence of SVS, DWI lesion volume, NIHSS on admission, TICI score and TOAST classification were entered into multivariate regression. The results presented in Table III revealed that besides NIHSS on admission and age, the presence of SVS was an independent factor to predict poor outcome (OR, 3.390; 95\% CI, 1.122-10.240; $\mathrm{P}=0.030)$.

In the 43 patients with SVS, proximal M1 SVS was seen in $18(41.9 \%)$ patients; distal M1 SVS in 10 (23.3\%) and distal MCA SVS in 15 (34.9\%). At 3 months after stroke, 18 patients achieved a favorable outcome, while the outcome was poor in 25 patients. Univariate analysis of the variables between SVS positive patients with and without favorable outcome are listed in Table IV. Patients with a poor outcome not only had larger infarction volumes on DWI [92.44 (28.62-245.91) vs. 27.18 (11.82-99.93) $\mathrm{ml} ; \mathrm{P}=0.016]$ and higher NIHSS scores on admission [17.6 \pm 8.12 vs. $6.39 \pm 3.99 ; \mathrm{P}<0.001]$, but also a higher rate of SVS located at the proximal M1 $(\mathrm{P}=0.044)$ and a greater SVS length [19.7 (13.6-33.75) vs. 10.3 (8.1-20.98) mm; $\mathrm{P}=0.017]$. Finally, NIHSS on admission, DWI lesion volume, the location of SVS and the length of SVS were subjected to multivariate regression analysis. However, only NIHSS on admission was an independent predictor of poor outcome in this model (OR, 1.341; 95\% CI, 1.123-1.602; P=0.001; Table III).

\section{Discussion}

The present study demonstrated that the presence of SVS was an independent predictor of poor outcome for patients with 
Table I. Clinical characteristics and radiological parameters of patients with and without SVS.

\begin{tabular}{|c|c|c|c|}
\hline Variable & $\operatorname{SVS}(+)(n=43)$ & $\operatorname{SVS}(-)(n=73)$ & P-value \\
\hline Age (years) & $60.35 \pm 13.21$ & $59.66 \pm 14.31$ & $0.796^{\mathrm{a}}$ \\
\hline Sex, female & $10(23.26)$ & $27(36.99)$ & $0.125^{\mathrm{a}}$ \\
\hline Time to MR imaging (h) & $38(24-50)$ & $40(24-60)$ & $0.513^{\mathrm{b}}$ \\
\hline Time to treatment $(\mathrm{h})$ & $24(9-36)$ & $24(15-48)$ & $0.114^{\mathrm{b}}$ \\
\hline Systolic BP (mm Hg) & $149.72 \pm 23.59$ & $137.74 \pm 17.29$ & $0.005^{\mathrm{a}}$ \\
\hline Blood glucose (mmol/l) & $5.56(4.83-6.31)$ & $5.34(4.44-6.80)$ & $0.424^{\mathrm{b}}$ \\
\hline \multicolumn{4}{|l|}{ Stroke risk factors } \\
\hline Hypertension & $23(53.49)$ & $35(47.95)$ & $0.564^{\mathrm{a}}$ \\
\hline Hyperlipidemia & $19(44.19)$ & $29(39.73)$ & $0.522^{\mathrm{a}}$ \\
\hline Diabetes mellitus & $12(27.91)$ & $15(20.55)$ & $0.365^{\mathrm{a}}$ \\
\hline Atrial fibrillation & $4(9.3)$ & $3(4.1)$ & $0.465^{\mathrm{a}}$ \\
\hline Hyperhomocysteinemia & $27(62.80)$ & $36(49.32)$ & $0.159^{\mathrm{a}}$ \\
\hline Smoking & $12(27.91)$ & $27(36.99)$ & $0.317^{\mathrm{a}}$ \\
\hline TOAST type & & & $0.062^{\mathrm{a}}$ \\
\hline Large artery atherosclerosis & 38 & 60 & \\
\hline Cardioembolism & 5 & 5 & \\
\hline Other etiologies & 0 & 8 & \\
\hline Infarct side, right & $22(51.2)$ & $38(52.05)$ & $0.926^{\mathrm{a}}$ \\
\hline Grade of TICI & $0(0-1)$ & $2(1-3)$ & $<0.001^{\mathrm{b}}$ \\
\hline Location of stenosis & & & $0.032^{\mathrm{a}}$ \\
\hline ICA & 6 & 14 & \\
\hline Proximal MCA & 29 & 35 & \\
\hline Distal MCA & 8 & 13 & \\
\hline Normal arteries & 0 & 11 & \\
\hline DWI lesion volume (ml) & $65.08(21.43-140.96)$ & $12.96(4.71-33.52)$ & $<0.001^{\mathrm{b}}$ \\
\hline NIHSS on admission & $12(5-20)$ & $5(2-8)$ & $<0.001^{\mathrm{b}}$ \\
\hline Favorable outcome & $18(41.9)$ & $58(79.5)$ & $<0.001^{\mathrm{a}}$ \\
\hline
\end{tabular}

${ }^{a}$ Independent samples t-test or $\chi^{2} ;{ }^{b}$ Wilcoxon rank sum test. Values are expressed as the mean \pm standard deviation, median $(25,75$ th percentile $)$ or $\mathrm{n}(\%)$. BP, blood pressure; DWI, diffusion-weighted imaging; ICA, internal carotid artery; MR, magnetic resonance; MCA, middle cerebral artery; mRS, modified Rankin scale; NIHSS, National Institutes of Health Stroke Scale; SVS, susceptibility vessel sign; TICI, thrombolysis in cerebral infarction; TOAST, Trial of Org 10172 in Acute Stroke Treatment.

acute anterior circulation stroke. For patients with SVS, only NIHSS on admission but not the location and length of SVS was a predictive factor of poor outcome at 3 months after stroke.

Magnetic susceptibility effects of the deoxygenated hemoglobin in the red thrombus result in a hypointense signal on $\mathrm{T} 2 *$ imaging. Cho et al (4) defined these hypointense signals within the vascular cisterns on T2* as 'SVS'. The GRE SVS may reflect the thrombus composition. Hemoglobin desaturation from oxyhemoglobin to deoxyhemoglobin occurs within a few h. Thus, the GRE SVS is present in older thrombi. Numerous studies have explored the predictive value of SVS regarding early recanalization or outcome for patients with acute stroke who had received reperfusion therapy. The presence of SVS was reported to not be associated with recanalization or outcome, but its location such as the proximal M1 $(7,8,17)$, its clot burden score (CBS) such as T2*-CBS >6 (10) and its morphology such as angle and length (11) were all found to have a predictive value regarding recanalization or outcome after t-PA therapy (17).

To the best of our knowledge, the present study was the first to investigate the association of SVS with the outcome for acute stroke patients who had not received any reperfusion therapy. The results demonstrated that in contrast to factors such as age and NIHSS on admission, SVS was also an independent factor to predict poor outcome 3 months after stroke. The following reasons may explain this phenomenon. First, SVS reflects red thrombi composed of erythrocyte-rich material (4). Patients with SVS have higher NIHSS scores and larger DWI infarction volumes. The initial neurological severity has an adverse impact on the clinical outcome. Furthermore, proximal M1 SVS, SVS with T2*-CBS $>6$ and longer SVS are resistant to intravenous thrombolysis $(6,10,11)$; in other words, distal MCA SVS, SVS with T2*-CBS $<6$, shorter SVS are relatively easy to resolve and patients with such presentations are more likely to benefit from reperfusion therapy. At present, as none of the 
Table II. Univariate analysis of the variables between patients with and without favorable outcome.

\begin{tabular}{|c|c|c|c|}
\hline Variable & $\begin{array}{c}\mathrm{mRS} \text { score }>2 \\
\quad(\mathrm{n}=40)\end{array}$ & $\begin{array}{c}\mathrm{mRS} \text { score } \leq 2 \\
\quad(\mathrm{n}=76)\end{array}$ & P-value \\
\hline Age (years) & $65.35 \pm 12.04$ & $57.05 \pm 13.96$ & $0.002^{\mathrm{a}}$ \\
\hline Sex, female & $15(37.5)$ & $22(28.9)$ & $0.348^{\mathrm{a}}$ \\
\hline Time to MR imaging (h) & $40(25.25-59)$ & $36(24-58.5)$ & $0.409^{\mathrm{b}}$ \\
\hline Time to treatment $(\mathrm{h})$ & $25.5(16.5-48)$ & $24(8-47)$ & $0.115^{\mathrm{b}}$ \\
\hline Systolic BP (mm Hg) & $140.5(130.5-156)$ & $138(128.25-153.75)$ & $0.221^{\mathrm{b}}$ \\
\hline Blood glucose (mmol/l) & $5.74(4.76-7.68)$ & $5.34(4.44-6.41)$ & $0.113^{\mathrm{b}}$ \\
\hline \multicolumn{4}{|l|}{ Stroke risk factors } \\
\hline Hypertension & $23(57.5)$ & $35(46.05)$ & $0.271^{\mathrm{a}}$ \\
\hline Hyperlipidemia & $20(50)$ & $28(36.8)$ & $0.171^{\mathrm{a}}$ \\
\hline Diabetes mellitus & $10(25)$ & $17(22.4)$ & $0.75^{\mathrm{a}}$ \\
\hline Atrial fibrillation & $6(15)$ & $1(1.3)$ & $0.003^{\mathrm{a}}$ \\
\hline Hyperhomocysteinemia & $23(57.5)$ & $40(52.6)$ & $0.617^{\mathrm{a}}$ \\
\hline Smoking & $10(25)$ & $29(38.2)$ & $0.154^{\mathrm{a}}$ \\
\hline TOAST type & & & $0.029^{\mathrm{a}}$ \\
\hline Large artery atherosclerosis & 34 & 64 & \\
\hline Cardioembolism & 6 & 4 & \\
\hline Other etiologies & 0 & 8 & \\
\hline Infarct side, right & $22(55)$ & $38(50)$ & $0.608^{\mathrm{a}}$ \\
\hline Grade of TICI & $1(0-2)$ & $2(0-2)$ & $0.043^{\mathrm{b}}$ \\
\hline Location of stenosis & & & $0.543^{\mathrm{a}}$ \\
\hline ICA & 8 & 12 & \\
\hline Proximal MCA & 6 & 15 & \\
\hline Distal MCA & 24 & 40 & \\
\hline Normal arteries & 2 & 9 & \\
\hline DWI lesion volume (ml) & $39.27(17.24-154.47)$ & $13.53(6.60-44.69)$ & $0.001^{\mathrm{b}}$ \\
\hline NIHSS on admission & $13.5(7.25-22.75)$ & $5(2-8)$ & $<0.001^{\mathrm{b}}$ \\
\hline SVS & $25(62.5)$ & $18(23.7)$ & $<0.001^{\mathrm{a}}$ \\
\hline
\end{tabular}

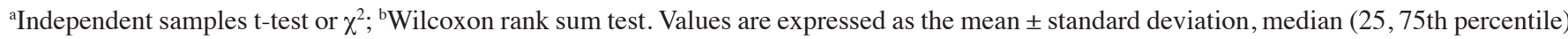
or n (\%). BP, blood pressure; DWI, diffusion-weighted imaging; ICA, internal carotid artery; MCA, middle cerebral artery; mRS, modified Rankin ccale; NIHSS, National Institutes of Health Stroke Scale; SVS, susceptibility vessel sign; TICI, thrombolysis in cerebral infarction; TOAST, Trial of Org 10172 in Acute Stroke Treatment; MR, magnetic resonance.

Table III. Multivariate logistic regression analysis for factors associated with an $\mathrm{mRS}$ score $>2$.

\begin{tabular}{lllr}
\hline Factor & OR & $95 \%$ CI & P-value \\
\hline For all enrolled patients & & & \\
$\quad$ Age & 1.085 & $1.036-1.136$ & 0.001 \\
Entry NIHSS & 1.278 & $1.145-1.426$ & $<0.001$ \\
SVS & 3.39 & $1.122-10.240$ & 0.03 \\
For patients with SVS & & & \\
$\quad$ Entry NIHSS & 1.341 & $1.123-1.602$ & 0.001 \\
\hline
\end{tabular}

CI, confidence interval; mRS, modified Rankin Scale; OR, odds ratio; SVS, susceptibility vessel sign; NIHSS, National Institutes of Health Stroke Scale. patients had received t-PA therapy, patients with distal MCA SVS or shorter SVS did not benefit from reperfusion treatment, so their outcome increased the probability of poor prognosis for patients with SVS.

Certain characteristics of SVS, such as proximal M1 location and the length were negative predictors regarding recanalization and clinical outcome for patients who received intravenous thrombolysis $(6,8,10,11)$ or intra-arterial intervention (12). However, the association of these parameters with the outcome for patients not subjected to thrombolysis had remained elusive. The present study found that in patients with SVS, the outcome was more favorable if it was not located at the proximal M1 SVS and if SVS had a shorter length. However, multivariate regression analysis revealed that none of them was an independent predictor regarding outcome. As mentioned above, an SVS at the proximal M1 represents a 
Table IV. Univariate analysis of the variables between SVS positive patients with and without favorable outcome.

\begin{tabular}{|c|c|c|c|}
\hline Variable & $\begin{array}{c}\mathrm{mRS} \text { score }>2 \\
\quad(\mathrm{n}=25)\end{array}$ & $\begin{array}{c}\text { mRS score } \leq 2 \\
\quad(n=18)\end{array}$ & P-value \\
\hline Age (years) & $63.52 \pm 12.04$ & $55.94 \pm 13.82$ & $0.063^{\mathrm{a}}$ \\
\hline Time to MR imaging (h) & $39.84 \pm 20.16$ & $33.11 \pm 17.11$ & $0.258^{\mathrm{a}}$ \\
\hline Time to treatment $(\mathrm{h})$ & $27.68 \pm 20.57$ & $20.06 \pm 13.93$ & $0.155^{\mathrm{a}}$ \\
\hline Systolic BP (mm Hg) & $154.4 \pm 23.54$ & $143.22 \pm 22.71$ & $0.127^{\mathrm{a}}$ \\
\hline Blood glucose (mmol/l) & $5.82(4.85-7.59)$ & $5.37(4.59-5.83)$ & $0.184^{\mathrm{b}}$ \\
\hline \multicolumn{4}{|l|}{ Stroke risk factors } \\
\hline Hypertension & $13(52)$ & $10(55.6)$ & $0.818^{\mathrm{a}}$ \\
\hline Hyperlipidemia & $11(44)$ & $8(44.4)$ & $0.977^{\mathrm{a}}$ \\
\hline Diabetes mellitus & $6(24)$ & $6(33.3)$ & $0.501^{\mathrm{a}}$ \\
\hline Atrial fibrillation & $4(16)$ & $0(0)$ & $0.211^{\mathrm{a}}$ \\
\hline Hyperhomocysteinemia & $16(64)$ & $11(61.1)$ & $0.847^{\mathrm{a}}$ \\
\hline Smoking & $6(24)$ & $6(33.3)$ & $0.501^{\mathrm{a}}$ \\
\hline TOAST type & & & $1.0^{\mathrm{a}}$ \\
\hline Large-artery atherosclerosis & 23 & 17 & \\
\hline Cardioembolism & 2 & 1 & \\
\hline Infarct side, right & $14(56)$ & $8(44)$ & $0.661^{\mathrm{a}}$ \\
\hline Grade of TICI & $0(0-1)$ & $0(0-1.25)$ & $0.382^{b}$ \\
\hline Location of stenosis & & & $0.818^{\mathrm{a}}$ \\
\hline ICA & 4 & 2 & \\
\hline Proximal MCA & 17 & 12 & \\
\hline Distal MCA & 4 & 4 & \\
\hline DWI lesion volume (ml) & $92.44(28.62-245.91)$ & 27.18 (11.82-99.93) & $0.016^{\mathrm{b}}$ \\
\hline Initial NIHSS & $17.6 \pm 8.12$ & $6.39 \pm 3.99$ & $<0.001^{\mathrm{a}}$ \\
\hline Location of SVS & & & $0.040^{\mathrm{a}}$ \\
\hline Proximal M1 & 14 & 4 & \\
\hline Distal M1 & 3 & 7 & \\
\hline Distal MCA & 8 & 7 & \\
\hline Length of SVS (mm) & $19.7(13.6-33.75)$ & $10.3(8.1-20.98)$ & $0.017^{b}$ \\
\hline
\end{tabular}

larger thrombus compared with the other distal thrombi with SVS, and a greater length of the SVS is indicative of a larger thrombus. Therefore, SVS that are longer and located at the proximal M1 are difficult to resolve by t-PA (8). By contrast, patients whose SVS is shorter or located at the distal M1 or MCA are more likely to achieve recanalization and have a favorable outcome. In the present study, as patients with small thrombi, such as SVS with a short length located at the distal MCA, did not benefit from reperfusion therapy, these parameters had no obvious predictive value regarding clinical outcome.

The results of the present study revealed that the TOAST category of patients with SVS was mostly cardioembolic stroke or large artery disease. None of the patients with SVS had any other etiologies of stroke, such as moyamoya disease or cryptogenic stroke. As previous studies demonstrated that the location, burden, morphology and length of the SVS were associated with no recanalization or poor outcome after intravenous thrombolysis or mechanical thrombectomy with stent retrievers, secondary prevention such as anti-thrombosis or anti-coagulation therapy to prevent the occurrence of SVS is important.

The present study supplemented previous studies, which assessed the association between SVS and recanalization or outcome for acute stroke patients who received reperfusion therapy. Of note, the present study had several limitations. First, the sample size was small. Furthermore, it was a retrospective study and certain patients were lost to follow-up. This may have contributed to selection bias. Third, sequence SWI was sensitive to motion artifacts, which added difficulty to the identification of SVS in certain patients.

In conclusion, the presence of SVS was found to be an independent factor to predict poor outcome for patients with acute stroke who had not received reperfusion therapy. The 
location and length of SVS were not predictors of clinical outcome for such patients.

\section{Acknowledgements}

The present study was supported by the Science and Technology Project of Henan Province (grant no. 162102310295).

\section{References}

1. Somford DM, Nederkoorn PJ, Rutgers DR, Kappelle LJ, Mali WP and van der Grond J: Proximal and distal hyperattenuating middle cerebral artery signs at CT: Different prognostic implications. Radiology 223: 667-671, 2002.

2. Schellinger PD, Chalela JA, Kang DW, Latour LL and Warach S: Diagnostic and prognostic value of early MR Imaging vessel signs in hyperacute stroke patients imaged $<3 \mathrm{~h}$ and treated with recombinant tissue plasminogen activator. AJNR Am J Neuroradiol 26: 618-624, 2005.

3. Flacke S, Urbach H, Keller E, Träber F, Hartmann A, Textor J, Gieseke J, Block W, Folkers PJ and Schild HH: Middle cerebral artery (MCA) susceptibility sign at susceptibility-based perfusion MR imaging: Clinical importance and comparison with hyperdense MCA sign at CT. Radiology 215: 476-482, 2000.

4. Cho KH, Kim JS, Kwon SU, Cho AH and Kang DW: Significance of susceptibility vessel sign on $\mathrm{T} 2 *$-weighted gradient echo imaging for identification of stroke subtypes. Stroke 36: 2379-2383, 2005.

5. Park MG, Yoon CH, Baik SK and Park KP: Susceptibility vessel sign for intra-arterial thrombus in acute posterior cerebral artery infarction. J Stroke Cerebrovasc Dis 24: 1229-1234, 2015.

6. Kimura K, Iguchi Y, Shibazaki K, Watanabe M, Iwanaga T and Aoki J: M1 susceptibility vessel sign on T2* as a strong predictor for no early recanalization after IV-t-PA in acute ischemic stroke. Stroke 40: 3130-3132, 2009.

7. Aoki J, Kimura K, Shibazaki K, Saji N, Uemura J, Sakamoto Y and Nagai K: The susceptibility vessel sign at the proximal M1: A strong predictor for poor outcome after intravenous thrombolysis. J Neurol Sci 348: 195-200, 2015.

8. Aoki J, Kimura K, Shibazaki K, Sakamoto Y, Saji N and Uemura J: Location of the susceptibility vessel sign on T2*-weighted MRI and early recanalization within 1 hour after tissue plasminogen activator administration. Cerebrovasc Dis Extra 3: 111-120, 2013
9. Aoki J, Kimura K, Shibazaki K, Saji N, Uemura J, Sakamoto Y and Nagai K: The susceptibility vessel sign at the proximal M1: A strong predictor for poor outcome after intravenous thrombolysis. J Neurol Sci 348: 195-200, 2015.

10. Legrand L, Naggara O, Turc G, Mellerio C, Roca P, Calvet D, Labeyrie MA, Baron JC, Mas JL, Meder JF, et al: Clot burden score on admission T2*-MRI predicts recanalization in acute stroke. Stroke 44: 1878-1884, 2013.

11. Yan S, Hu H, Shi Z, Zhang X, Zhang S, Liebeskind DS and Lou M: Morphology of susceptibility vessel sign predicts middle cerebral artery recanalization after intravenous thrombolysis. Stroke 45: 2795-2797, 2014.

12. Soize S, Batista AL, Rodriguez Regent C, Trystram D, Tisserand M, Turc G, Serre I, Ben Hassen W, Zuber M, Calvet D, et al: Susceptibility vessel sign on T2* magnetic resonance imaging and recanalization results of mechanical thrombectomy with stent retrievers: A multicentre cohort study. Eur J Neurol 22: 967-972, 2015.

13. Yan S, Hu H, Shi Z, Zhang X, Zhang S, Liebeskind DS and Lou M: Morphology of susceptibility vessel sign predicts middle cerebral artery recanalization after intravenous thrombolysis. Stroke 45: 2795-2797, 2014.

14. Adams HP Jr, Bendixen BH, Kappelle LJ, Biller J, Love BB, Gordon DL and Marsh EE III: Classification of subtype of acute ischemic stroke. Definitions for use in a multicenter clinical trial. TOAST. Trial of Org 10172 in acute stroke treatment. Stroke 24:35-41, 1993.

15. Naggara O, Raymond J, Domingo Ayllon M, Al-Shareef F, Touzé E, Chenoufi M, Gerber S, Mellerio C, Zuber M, Meder JF, et al: T2* 'susceptibility vessel sign' demonstrates clot location and length in acute ischemic stroke. PLoS One 8: e76727, 2013.

16. Higashida RT, Furlan AJ, Roberts H, Tomsick T, Connors B, Barr J, Dillon W, Warach S, Broderick J, Tilley B, et al: Trial design and reporting standards for intra-arterial cerebral thrombolysis for acute ischemic stroke 34: e109-e137, 2003.

17. Apoil M, Turc G, Tisserand $M$, Calvet $D$, Naggara $O$, Domigo V, Baron JC, Oppenheim C and Touzé E: Clinical and magnetic resonance imaging predictors of very early neurological response to intravenous thrombolysis in patients with middle cerebral artery occlusion. J Am Heart Assoc 2: e000511, 2013. 